\title{
A laboratory study of spontaneous platelet aggregation
}

\author{
I. FRIEDLANDER ${ }^{1}$, I. J. Y. COOK, C. HAWKEY, AND C. SYMONS \\ From New End and the Royal Free Hospitals, London, and the Nuffield Institute of Comparative Medicine, \\ The Zoological Society of London
}

SYNOPSIS During studies on platelet aggregation using the EEL platelet aggregation meter, $8 \%$ of the individuals tested were found to have platelets which aggregated spontaneously when citrated, platelet-rich plasma was stirred at $37^{\circ} \mathrm{C}$. The EEL aggregation meter differs from other machines in that it incorporates a vertical stirrer which subjects platelets to greater mechanical force. When using this machine it is suggested that spontaneous platelet aggregation is related to increased mechanical fragility of the platelets and low levels of plasma ADP-inhibitor.

Platelets circulate in the bloodstream as separate particles and this property is retained in correctly anticoagulated, carefully collected blood. Born and Cross (1963), using a magnetic stirrer, have reported that citrated, platelet-rich plasma can be stirred for one hour without significant aggregation, and O'Brien, Heywood, and Heady (1966) have found that only a small degree of aggregation sometimes occurs when citrated platelet-rich plasma is stirred at $37^{\circ} \mathrm{C}$.

During studies on platelet aggregation using the EEL platelet aggregation meter (Cook and Symons, 1966), marked spontaneous platelet aggregation was noted in some individuals. Subsequently the test for spontaneous platelet aggregation was carried out on all samples, and of 244 subjects tested, 18 exhibited this phenomenon. This paper presents the results of investigations designed to determine whether spontaneous platelet aggregation is an artefact induced by the experimental system or whether it is an intrinsic property of the plasma or platelets.

\section{Materials and Methods}

Blood samples were collected from normal volunteers and patients with ischaemic heart disease. Individuals from both groups whose platelets showed spontaneous aggregation were studied further. Individuals whose platelets did not exhibit this phenomenon were used as controls.

${ }^{1}$ Present address: Institute of Child Health, Guilford Street, London, wC1.

Received for publication 24 August 1970.
Polypropylene pipettes and disposable polystyrene containers were used throughout the experiments. Using disposable plastic syringes, $18 \mathrm{ml}$ of blood was obtained by clean venepuncture and mixed with $2 \mathrm{ml}$ of $3.8 \%$ trisodium citrate. Platelet-rich plasma was prepared immediately by centrifugation at room temperature for 10 minutes at $1,000 \mathrm{rpm}$. Volumes each of $1 \mathrm{ml}$ were placed in disposable polystyrene tubes. Platelets were counted by the method of Brecher and Cronkite (1950).

Platelet aggregation was measured by an adaptation of the method of Born (1962), using the EEL platelet aggregation meter (Cook and Symons, 1966). This apparatus measures changes in optical density of platelet-rich plasma at $37^{\circ} \mathrm{C}$ as platelets aggregate. After prewarming at $37^{\circ} \mathrm{C}$ for 90 seconds platelet-rich plasma to be tested for spontaneous aggregation was transferred to the reaction chamber of the aggregation meter and stirred by a flat-tipped stainless steel, vertical stirrer at a speed of 4,000 rpm unless otherwise stated. A tachometer was attached to the apparatus so that the speed of stirring could be determined. Optical density (OD) was recorded continuously by a potentiometric recorder at a sensitivity of $5 \mathrm{mV}$. The sensitivity of the recorder was adjusted to give the same initial reading for each sample. The difference between the initial OD and the OD after 10 minutes was expressed in arbitrary OD units. Spontaneous aggregation was considered to have occurred if there was a change in OD of 20 units or more in 10 minutes. The spontaneous aggregation time was measured as the time taken from the start of stirring to the point at which an OD change of 20 units was reached. Samples 
showing spontaneous aggregation were subjected to a further 20 minutes of stirring to determine if disaggregation occurred subsequently.

Sensitivity of platelets to aggregating agents was tested by stirring prewarmed samples, each of $1 \mathrm{ml}$, of platelet-rich plasma in the aggregation meter for 60 seconds before adding $0 \cdot 1 \mathrm{ml}$ of the test aggregating substance. Agents tested included $10^{-4} \mathrm{M}$ adrenaline, $10^{-4} \mathrm{M}$ noradrenaline, $10^{-4} \mathrm{M}$ 5-hydroxytryptamine (5HT), $6.25 \mu \mathrm{g} / \mathrm{ml}$ adenosine diphosphate (ADP), $0.25 \mathrm{NIH}$ units $/ \mathrm{ml}$ thrombin, and collagen extracted from calf tendon.

Plasma ADP-inhibitor was measured by the method of Cook and Symons (1966). Standard ADP was mixed with platelet-rich plasma and preincubated at $37^{\circ} \mathrm{C}$ without stirring for varying periods of time. The residual ADP activity was then measured by recording change in OD in the mixture on stirring. Individuals were classed as having 'high' ADPinhibitor levels if the OD change was less than 20 OD units after a preincubation time of four minutes. Those in which the change in OD was greater than 20 units after preincubation for four minutes were considered to have 'low' ADP-inhibitor levels.

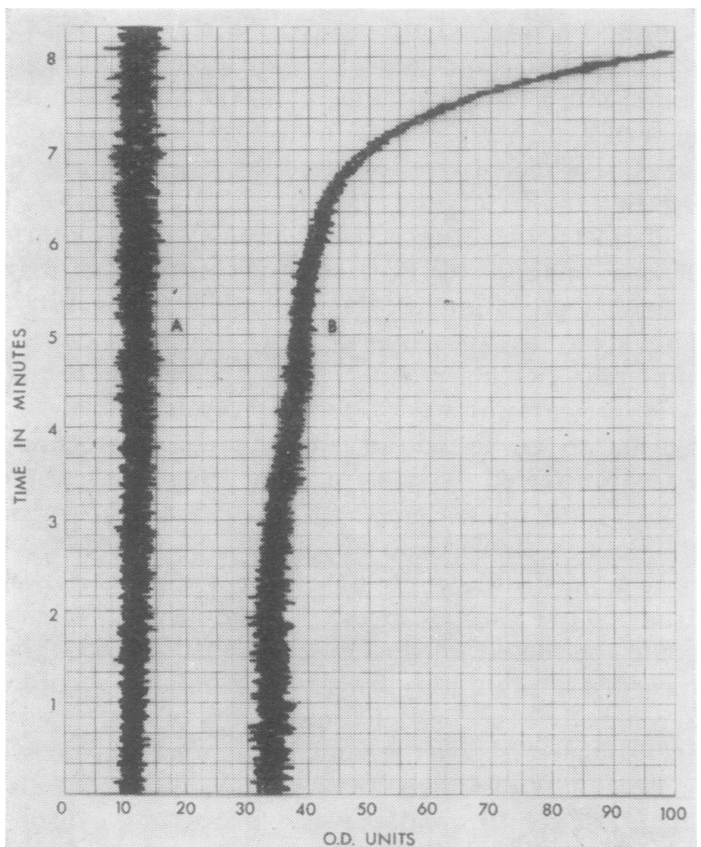

Fig. 1

\section{Results}

CHARACTERIZATION OF SPONTANEOUS

PLATELET AGGREGATION

Of 224 individuals studied, $18(8 \%)$ showed spon흔 taneous platelet aggregation. In repeated tests on? these subjects the finding was reproducible in $14^{n}$ $(80 \%)$. Platelets that aggregated spontaneously did not show subsequent disaggregation (Fig. 1).

Influence of platelet count

Spontaneous aggregation could only be demonstratect if the platelet concentration of the test platelet-riche plasma was greater than $320,000 / \mathrm{cmm}$ (Fig. 2)용 Above this concentration the degree of aggregationi was proportional to the platelet count (Fig. 2). $\mathrm{No}_{+}^{+}$ aggregation could be induced in control samples byi varying the platelet count.

\section{Influence of storage and temperature}

In samples tested immediately after collection the spontaneous platelet aggregation time was fromk five to 10 minutes. This time was shortened to threec to four minutes if the samples of platelet-rich plasmar

Fig. 1 Changes in optical density when platelet-rich plasma is stirred at 4,000 rpm without the addition of any aggregating agent. (A) No aggregation; (B) spontaneous aggregation.

Fig. 2 Influence of platelet concentration on spontaneous aggregation: (A) 280,000/cmm; (B) 325,000/cmm; (C) $375,000 / \mathrm{cmm}$; (D) $495,000 / \mathrm{cmm}$.

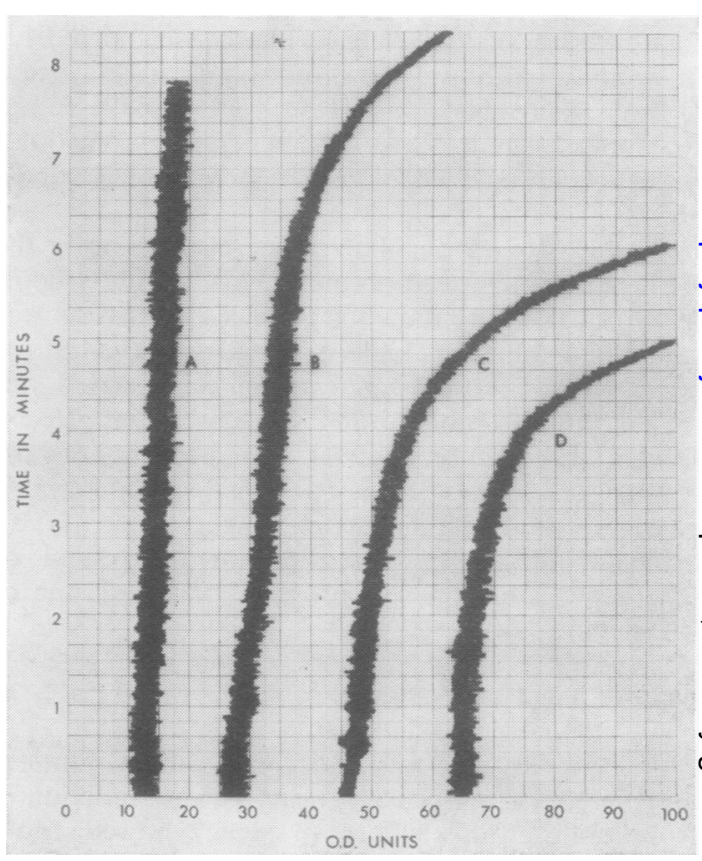

Fig. 2 


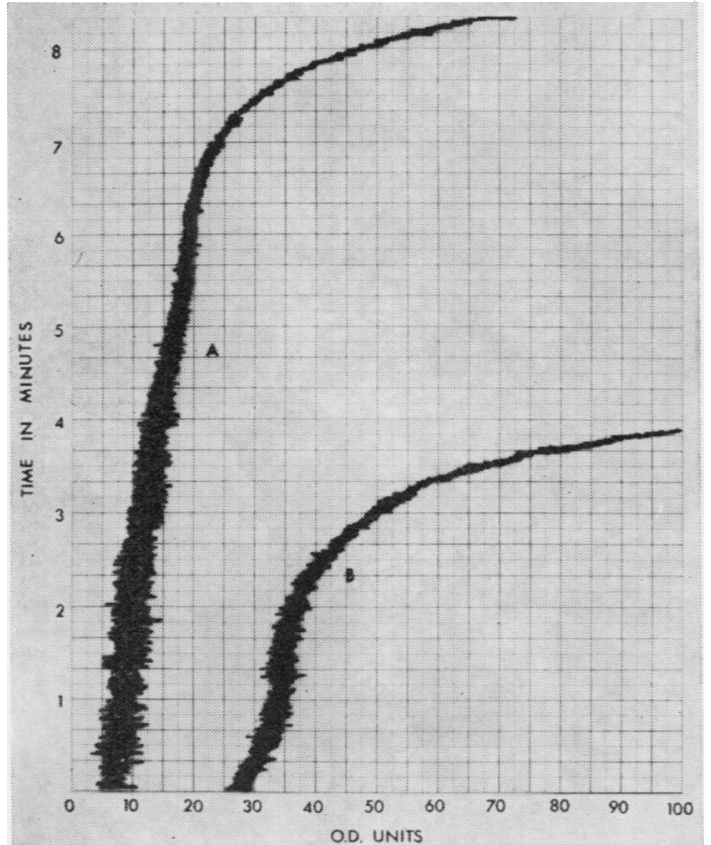

Fig. 3

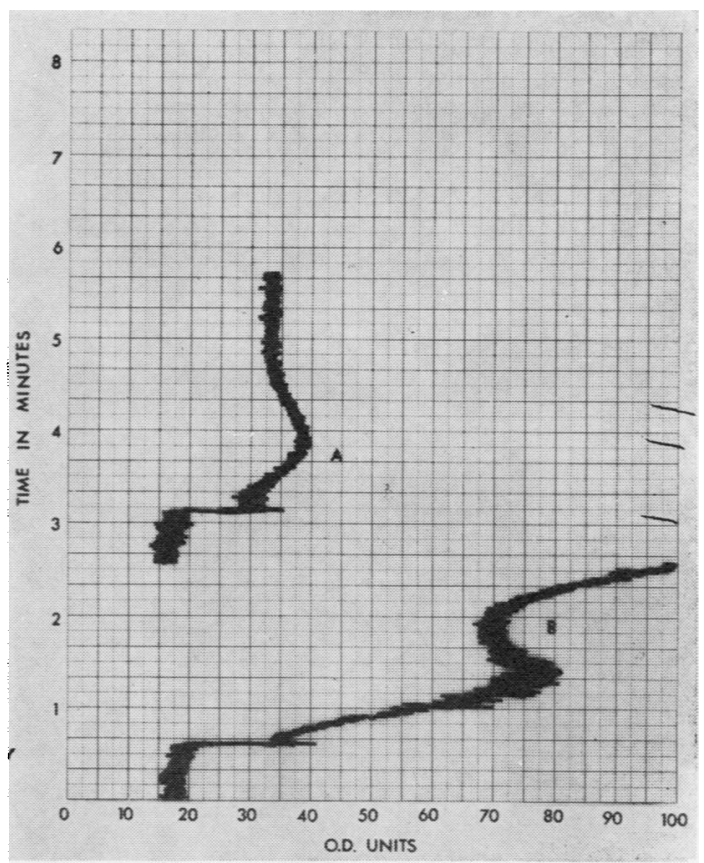

Fig. 4

Fig. 3 Effect of storage of plasma at room temperature $\left(20^{\circ} \mathrm{C}\right)$ on spontaneous aggregation: (A) Plasma tested immediately after separation. (B) Plasma tested after two hours at $20^{\circ} \mathrm{C}$.

Fig. 4 Platelet aggregation induced by addition of 0.25 N.I.H. units/ml of thrombin to platelet-rich plasma of (A) aggregation.

were left at room temperature for two hours before testing (Fig. 3). Spontaneous aggregation was preserved in platelet-rich plasma stored at $4^{\circ} \mathrm{C}$ for two hours but not if it was incubated at $37^{\circ} \mathrm{C}$ for two hours.

Although spontaneous platelet aggregation could be preserved at room temperature in platelet-rich plasma this property disappeared in whole blood left at room temperature for two hours or more before centrifugation and separation of platelet-rich plasma. These platelets, however, could still be, aggregated by adding ADP to the platelet-rich plasma.

\section{Influence of aggregating agents}

Platelets which aggregated spontaneously showed a greater sensitivity than normal on addition of ADP, 5HT, adrenaline, noradrenaline, collagen, or thrombin (Fig. 4). The plasma level of ADP-inhibitor was consistently low in these individuals, although not all subjects with low plasma ADP-inhibitor levels also exhibited spontaneous aggregation.
Properties of the supernatant plasma after spontaneous aggregation

Supernatant plasma from platelet-rich plasma in which spontaneous platelet aggregation had occurred aggregated the platelets in control platelet-rich plasma similarly to ADP (Fig. 5). However, plateletrich plasma separated from test subjects before spontaneous aggregation had been induced produced no aggregation of control platelets.

INFLUENCE OF EXPERIMENTAL CONDITIONS ON SPONTANEOUS AGGREGATION

Anticoagulant

Blood was collected from test and control individuals into three different batches of trisodium citrate obtained from different sources. The distribution of spontaneous aggregation was not altered.

\section{Contamination of apparatus}

To exclude the possibility that traces of an aggregating agent might be contaminating the apparatus, platelet-rich plasma from individuals showing 


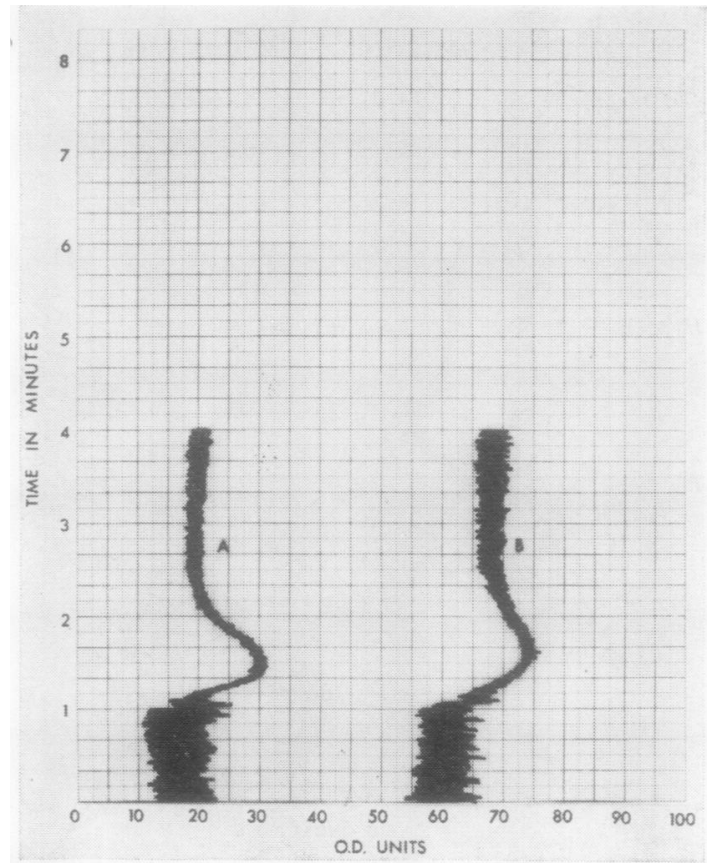

Fig. 5

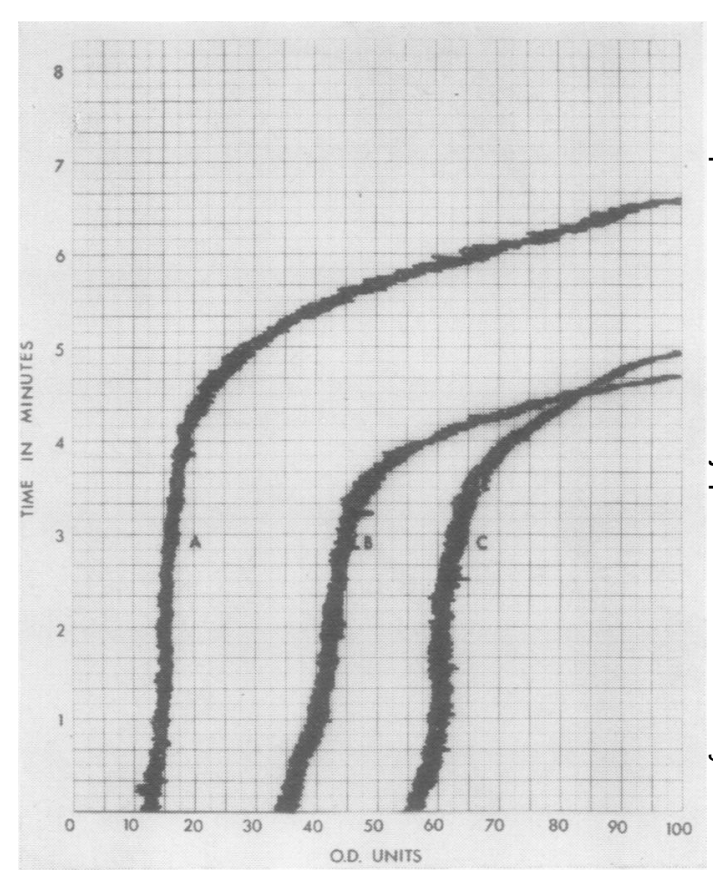

Fig. 6

Fig. 5 The effect on control platelet-rich plasma of addition of (A) supernatant plasma after spontaneous platelet aggregation and (B) ADP at a concentration of $6.25 \mu \mathrm{g} / \mathrm{ml}$.

Fig. 6 Influence of speed of stirring on spontaneous platelet aggregation: (A) 1,000 rpm; (B) 2,000 rpm; (C) 4,000 rpm.

spontaneous aggregation was retested using a new, siliconed stirrer, new tubes and pipettes. The pattern of spontaneous aggregation was not altered.

\section{Contamination of blood sample}

Substances known to cause platelet aggregation which might be present in the blood sample include ADP from disrupted red cells and thrombin produced by partial activation of the coagulation mechanism. The finding that spontaneous aggregation is present in platelet-rich plasma but disappears in whole blood stored for two hours at $37^{\circ} \mathrm{C}$ suggested that red cell ADP was not the cause of aggregation. Haemoglobin could not be demonstrated photometrically in these samples. The addition of heparin (0.05-5.0 units $/ \mathrm{ml}$ ) to platelet-rich plasma did not inhibit spontaneous aggregation, indicating that contamination with trace amounts of thrombin, insufficient to bring about overt clotting but enough to cause the platelets to aggregate, was not responsible for the phenomenon.

\section{Drug intake}

Drug intake could not be correlated with the occurrence of spontaneous platelet aggregation.
Age and sex

Spontaneous aggregation was found in subjects of both sexes. There was no obvious relationship. between the occurrence of spontaneous aggregation and age.

\section{Speed of stirring}

The maximum stirring speed which can be obtained with the EEL aggregation meter is $4,000 \mathrm{rpm}$. This은 speed, which has been routinely used for all the tests so far reported, is considerably faster than the maximum speed of $1,000 \mathrm{rpm}$ used by most othern experimenters in this field (Born, 1962; O'Brien, 1962).

Tests carried out on test platelet-rich plasma at different stirring speeds demonstrated that the speed of stirring affected the spontaneous aggregatione time (Fig. 6), the delay period before onset of spon-क taneous aggregation being from one to three minutes.? longer in samples stirred at $1,000 \mathrm{rpm}$ than ato $2,000 \mathrm{rpm}$ or faster. However, samples of platelet-rich plasma from all individuals in which spontaneous? aggregation was present when stirring was at $4,000 \mathrm{0}$ rpm also showed spontaneous aggregation wheng stirred at rates of $500 \mathrm{rpm}$ to $3,000 \mathrm{rpm}$. 


\section{Discussion}

Using the EEL platelet aggregation meter, marked spontaneous platelet aggregation occurred in $18(8 \%)$ out of 224 individuals tested. In $14(80 \%)$ of these it was present repeatedly in blood samples obtained over a period of several months.

Experiments described in this paper demonstrate that the phenomenon was not due to trace contamination of the apparatus with an aggregating agent, variation in the citrate used as anticoagulant, or differences in the number of platelets present in the platelet-rich plasma. Red cell ADP or thrombin, present as a result of partial activation of the coagulation mechanism, do not appear to contribute to the phenomenon.

The results obtained indicate that spontaneous aggregation in stirred citrated plasma is an inherent characteristic of the platelets of some individuals. Platelet-poor plasma from test individuals did not aggregate platelets in control platelet-rich plasma unless it was separated after spontaneous aggregation had occurred, suggesting that an aggregating agent is released from the test platelets during the process. Haslam (1964) has suggested that ADP forms the 'final common path' in the action of all substances known to cause platelet aggregation and, whatever the initiator of spontaneous aggregation, ADP released from the test platelets is probably the substance responsible for continuing the process.

Plasma ADP-inhibitor levels were low in all subjects showing spontaneous aggregation but a similar proportion of control subjects showed equally low levels. However, in no instance were high levels of ADP-inhibitor associated with spontaneous aggregation. Lack of ADP-inhibition could be responsible for persistence in the plasma of trace amounts of ADP but does not explain how the process of spontaneous aggregation is triggered.

One important difference between the EEL aggregation meter and other machines designed to measure platelet aggregation is that, instead of the conventional magnetic pea stirrer, a vertical stirrer is used which subjects the whole volume of plateletrich plasma to greater mechanical force. This may be powerful enough to disrupt some platelets, resulting in the release of their constituents, including intrinsic platelet ADP. This ADP, in individuals with low levels of plasma ADP-inhibitor, would thus be available to trigger the process of spontaneous platelet aggregation. Subsequent release of further platelet ADP which is known to occur during aggregation (Haslam, 1964) could amplify the reaction. Spontaneous platelet aggregation was only found in $8 \%$ of the subjects tested. These individuals may represent a fraction of the population in which the platelets are more than normally susceptible to breakdown by mechanical agitation and the plasma ADP-inhibitor level is low. High reactivity of the platelets of these individuals to added aggregating agents may enhance the response.

These findings suggest that the stirring mechanism used in the EEL aggregation meter renders this apparatus capable of distinguishing a population of individuals in whom the platelets have a high mechanical fragility and the plasma ADP-inhibitor level is low. Whether or not this situation could have any clinical significance is open to speculation. In this preliminary study no attempt has been made to take into account the full clinical status of the individuals in which spontaneous platelet aggregation has been found, but it should perhaps be restated that they were drawn from a series comprising patients with cardiovascular disease and overtly healthy controls. A further survey is required to determine if platelets with high mechanical fragility are involved in the aetiology of any disease states, but even if the phenomenon is shown to have no clinical significance, its influence on the results of platelet aggregation tests should be taken into account.

This study was supported by research grants from the North West Metropolitan Regional Hospital Board and the Medical Research Council.

\section{References}

Born, G. V. R. (1962). Aggregation of blood platelets by adenosine diphosphate and its reversal. Nature (Lond.), 194, 927-929.

Born, G. V. R., and Cross, M. J. (1963). The aggregation of blood platelets. J. Physiol. (Lond.), 168, 178-195.

Brecher, G., and Cronkite, E.P.(1950). Morphology and enumeration of human blood platelets. J. appl. Physiol., 3, 365-367.

Cook, I. J. Y., and Symons, C. (1966). Measurement of ADP-induced platelet aggregation and ADP-splitting enzymes in plasma. Lancet, 2, 623-624.

Haslam, R. J. (1964). Role of adenosine diphosphate in the aggregation of human blood-platelets by thrombin and by fatty acids. Nature (Lond.), 202, 765-768.

O'Brien, J. R. (1962). Platelet aggregation. II. Some results from a new method of study. J. clin. Path., 15, 452-455.

O'Brien, J. R., Heywood, J. B., and Heady, J. A. (1966). The quantitation of platelet aggregation induced by four compounds; a study in relation to myocardial infarction. Thrombos. Diathes. haemorrh. (Stutt.), 16, 752-767.

Please address request for reprints to C. H., Zoological Society of London, Regents Park, NW1. 